\title{
GENU VALGUM.
}

\section{Orthopaedics}

\section{Angelin Mersha}

CRRI - Intern, Chettinad Hospital And Research Institute, chettinad Academy Of

Dr. Venkatachalam.
K*

Research And Education,kelambakkam, Chengalpattu District, Pin - 603103.

Prof. And HOD, Department Of Orthopaedics,medical Superintendent, Chettinad Hospital And Research Institute, Chettinad Academy Of Research And Education, Kelambakkam, Chengalpattu District, Pin - 603103. *Corresponding Author

Dr. Arun Kumar C Assisstant Professor,Department Of Orthopaedics,chettinad Hospital And Research Dr. Arun Kumar C Institute,chettinad Academy Of Research And Education,kelambakkam, Chengalpattu District, Pin - 603103.

MS (ORTHO) PG,Department Of Orthopaedics,chettinad Hospital And Research Dr.Vibishek Raj .P Institute, Chettinad Academy Of Research And Education, Kelambakkam, Chengalpattu District, Pin-603103.

\section{KEYWORDS}

Genu Valgum, Knock Knees, Hemi-Epiphysiodesis

\section{INTRODUCTION :}

Genu valgum or "knock knees" are deformities of the lower limb that occur in the coronal plane. ${ }^{[1]}$ Though most patients are asymptomatic and are fully functional, these conditions are sometimes indicated by a flat feet and medial aspect of foot and knee pain. The normal alignment of the knee joint is at a minimal valgus position, attained at around 7 years of age, giving the knee the stability required for mobilization. However, in the paediatric age group, the alignment of the knee joint changes from physiological genu valgum by 2 years to varus at about 7 years of age..$^{[2]}$

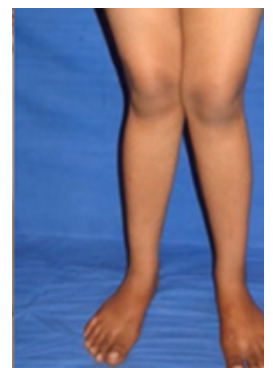

Fig 1 : Patient with genu valgum deformity.

\section{ETIOLOGY :}

1. Bilateral genu valgum ${ }^{[2]}$ :

(a) Physiologic genu valgum

(b) Skeletal dysplasias - (Morquio syndrome, Spondylo-epiphyseal dysplasia, Chondro-ectodermal dysplasia)

(c) Metabolic bone diseases

(d) Lysosomal storage diseases

2. Unilateral genu valgum ${ }^{[2]}$ :

(a) Post traumatic

(b) Tumors

(c) Infection

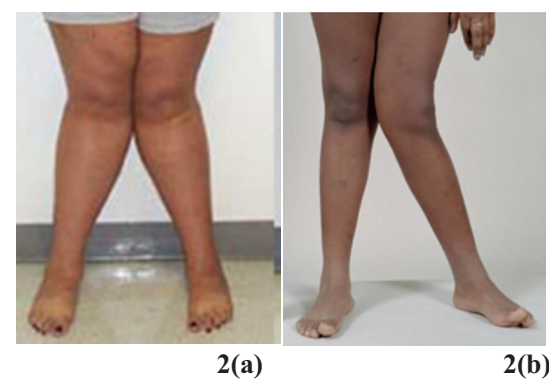

Fig 2(a) : Bilateral genu valgum.

Fig 2(b) : Unilateral genu valgum.

\section{EPIDEMIOLOGY :}

The main complaints in these paediatric patients, at presentation, is a cosmetic deformity. Genu valgum, is more noticeable in children, at about 3-5 years of age. The primary etiology, in bilateral cases is, physiologic genu varum, however abnormal skeletal growth can occur due to spondylo-epiphyseal dysplasia and chondroectodermal dysplasia (Ellis van Creveld syndrome), metabolic bone diseases such as (no,) rickets (renal osteodystrophy and hypophosphatemic rickets) and lysosomal storage disease such as Morquio syndrome may be the reason for pathological genu valgum. ${ }^{[2]}$ The deformity is more commonly present at the distal femur, compared to proximal tibia. ${ }^{[1]}$

Other relatively rare causes include radiation, infection and tumors (osteochondromas, multiple hereditary exostoses, fibrous dysplasia etc). ${ }^{[3]}$

\section{EVALUATION :}

Gait and rotational profile analysis are important to spot the cause and the differences behind the knocked knees. When a patient stands with the medial position of the femoral condyle touching each other, the distance between the medial maleoli of the opposite legs, called the inter-malleolar distance is usually less than $8 \mathrm{cms}$. The diagnosis is confirmed, when the finding is contradicted. ${ }^{[1]} \mathrm{X}$-rays are not indicated in children, in the physiologic valgus phase. ${ }^{[2]}$ They are indicated in asymmetrical findings; i.e :

(I) In an age group beyond which is expected of physiologic changes.

(ii) Patients whose height falls below the tenth percentile for their age.

(iii) History of trauma.

(iv) History of infection.

Coronal plane angulation of the lower extremities can be analyzed by two methods either based on the deviation of the mid-point of the knee from the mechanical axis on the tibio-femoral angle. In normal coronal alignment, the mechanical axis passes through the mid point of the knee. There is lateral and medial deviation of the center of the knee with respect to the mechanical axis of the lower extremity in genu varus (bowed legs) and genu valgum (knocked knee), respectively. ${ }^{[3]}$

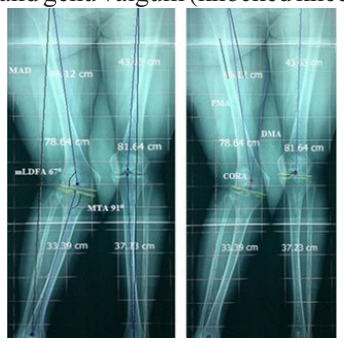

Fig 3 : X-ray of lower limbs showing anatomical axis and mechanical axis in a case of genu valgum. 
The tibio-femoral angle is the acute angle formed in the middle of longitudinal axes of the tibial and femoral shafts. At birth, there is 15 to 20 degrees of varus tibio-femoral angulation. As the child grows, this corrects to neutral, by about the age of 2 and between 10 to 15 degrees of valgus tibio-femoral angulation between ages 3 and 4 . At this point, the limb's valgus angulation, then starts to gradually decrease to approximately, 3-5 degrees of valgus by the age of 7 . This is the residual normal coronal plane angulation of the lower extremity, that will be carried to adulthood and should not increase. ${ }^{[4]}$

It is important, to determine whether the deformity is primarily originating from the femur or tibia. This is done by measuring the mechanical lateral distal femoral angle (angle between the femoral shaft and the mechanical axis of the femur) and medial proximal tibial angle (angle between the tibial plateau and mechanical axis of the tibia). The normal range of these angles is between 85 and 90 degrees. ${ }^{[5]}$

\section{TREATMENT PROTOCOL:}

(a) Observation is the first line of treatment if genu valgum is less than 15 degrees in child less than 6 years of age

(b) Medical management is the first-line treatment for angular deformities attributed to metabolic disorders.

(c) Hemi-epiphysiodesis is typically used to correct angular deformities.

Indications of medial sided hemi-epiphysiodesis about the distal femur and/or proximal tibia include $>15$ to 20 degrees in a child $<10$ years of age and if the mechanical axis falls on the lateral quadrant of the proximal tibia in a patient $>10$ years of age. Osteotomies about the distal femur are also used in the management of genu valgum. ${ }^{[6]}$

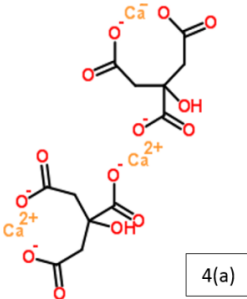

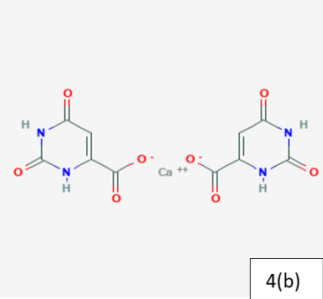

Fig 4(a): Molecular structure of calcium citrate. Fig 4(b) : Molecular structure of calcium orotate.

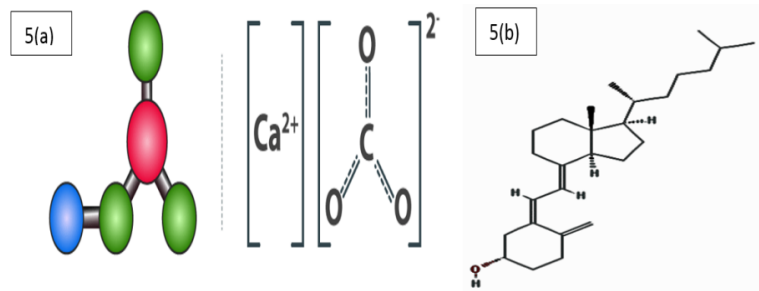

Fig 5(a): Molecular structure of calcium carbonate.

Fig 5(b) : Molecular structure of vitamin $D_{3}$ (Cholecalciferol)

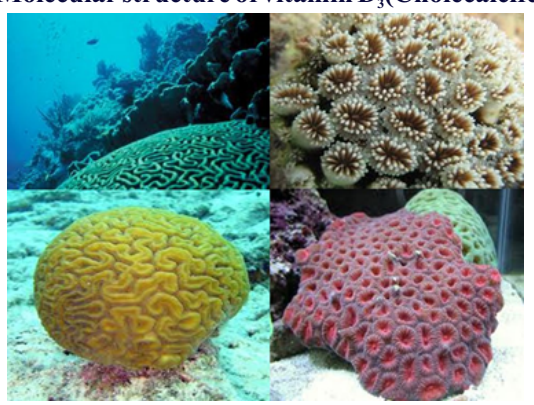

Fig 6 : Calcium derived from coral reef.

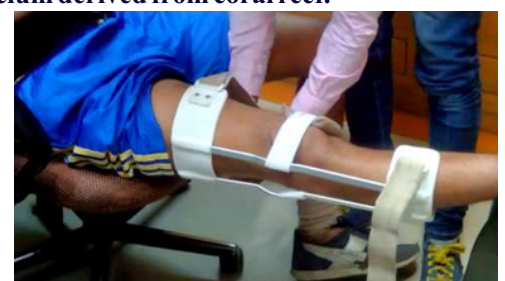

Fig 7 : Brace for genu valgum.

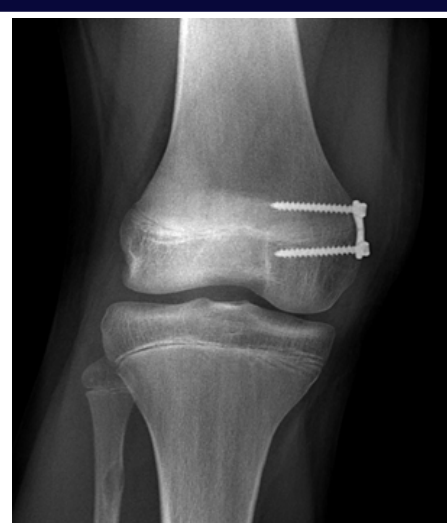

Fig 8 : Post-op X-ray after medial femoral epiphysiodesis on left side.

\section{CONCLUSION :}

Physiologic genu valgum almost always correct spontaneously. Pathologic genu valgum is usually associated with some metabolic bone diseases and it may improve, when the underlying disorder is remedied.$^{[7]}$ Lower femoral bone commonly accounts for pathological genu valgum. Rarely however, upper tibia may be the causative factor.

\section{REFERENCES:}

1. Patel M, Nelson R. Genu Valgum. In: StatPearls. Treasure Island (FL): StatPearls Publishing; June 13, 2020 .

2. Walker JL, Hosseinzadeh $\mathrm{P}$, White $\mathrm{H}$, et al. Idiopathic Genu Valgum and Its Association With Obesity in Children and Adolescents. J Pediatr Orthop. 2019;39(7):347-352. doi:10.1097/BPO.0000000000000971

3. PRETKIEWICZ-ABACJEW E: Knock knee and the gait of sixyear-old children. J Sports Med Phys Fitness 43: 156, 2003.

4. KRIVICKAS LS: Anatomical factors associated with overuse sports injuries. Sport Med 24: 132, 1997

5. COWAN DN, JONES BH, FRYKMAN PN, ET AL: Lower limb morphology and risk of overuse injury among male infantry trainees. Med Sci Sports Exerc 28: 945, 1996.

6. NYLAND J, SMITH S, BEICKMAN K, ET AL: Frontal plane knee angle affects dynamic postural control strategy during unilateral stance. Med Sci Sports Exerc 34 1150,2002

7. SGARLATO TE (ED): A Compendium of Podiatric Biomechanics, p 381, Californi College of Podiatric Medicine, San Francisco, March 1971. 